anticomplementary. Further work to confirm the nature of immune complexes associated with parvovirus B19 infection is warranted.

1 Anderson MJ, Pattison JR. The human parvovirus. (Brief review). Arch Virol 1984;82:137-48.

2 Keller ML. Human Parvovirus B19: Not just a paediatric problem. Emerg Med 1992;10:39-44.
3 Cohen BJ, Mortimer PP, Pereira MS. Diagnostic assays with monoclonal antibodies for the human serum parvovirus like virus (SPLV). $\mathcal{f}$ Hyg 1983;91:113-30.

4 Mori J, Field AM, Clewley JP, Cohen BJ. Dot blot hybridization assay of B19 virus DNA in clinical specimens. brin Microbiol 1989;27:459-64.

5 Joseph PR. Fifth Disease: the frequency of joint involvement in adults. NY State $\mathcal{F}$ Med 1986;86:560-3.

6 Porter DD. Aleutian Disease: A persistent parvovirus infection of mink with a maximal but ineffective host humoral immune response. Prog Med Virol 1986;33:42-60.

\title{
Expression of MHC class II antigens by placental villi: no relationship with villitis of unknown origin
}

\author{
T Y Khong
}

\begin{abstract}
The aim of the study was to determine whether immunoreactivity to major histocompatibility complex (MHC) class II antigens studied by immunohistochemistry could be used reliably to define villitis lesions in placenta. Eighteen placental sections with villitis and 32 without, as determined in a careful observer reproducibility study, were immunolabelled with a monoclonal antibody to monomorphic determinants of MHC class II antigens (CR3/43), using a standard avidin-biotin peroxidase technique. Placentas with villitis were found to express MHC class II antigens. However, some showed no immunoreactivity. Occasional villi unaffected by villitis, including those near placental infarcts, also expressed MHC class II antigens. The study therefore showed that immunohistochemistry cannot be used to define villitis of unknown aetiology. It provides further evidence of the difficulties that can arise when immunohistochemistry conflicts with previous light microscopy findings.

(f Clin Pathol 1995;48:494-495)
\end{abstract}

Keywords: Villitis of unknown aetiology, immunohistochemistry, MHC class II antigens, placental pathology.

Villitis of unknown aetiology (VUA) is associated with intrauterine growth retardation, preeclampsia, and stillbirth and may recur in subsequent pregnancies with similar associations. The essential feature of VUA is the accumulation of chronic inflammatory cells in the placental villous stroma. ${ }^{1}$ Because no recognised infectious agent has been identified, it has been theorised that VUA is a marker of maternal immune attack on the fetal allograft ${ }^{2}$ and a recent finding that these chronic inflammatory cells are of maternal origin would lend support to this suggestion. ${ }^{3}$ Expression of major histocompatibility complex (MHC) class II antigens by syncytiotrophoblast, macrophages, and vessels within placental villi has been cited as further evidence of immunopathology in the lesion and has been suggested as a marker for VUA, and been used to define the lesion. ${ }^{4-7}$ To test this hypothesis, immunohistochemistry was performed on a series of placental cases that were the subject of a study to assess observer reliability in diagnosing villitis using conventional criteria. ${ }^{8}$

\section{Methods}

Sequential $5 \mu \mathrm{m}$ sections from 50 formalin fixed paraffin embedded placentas that were used for an observer reliability study ${ }^{8}$ were rehydrated and later subjected to immunohistochemistry using a standard avidin-biotinhorseradish peroxidase technique with $3^{\prime}, 3^{\prime}$ diaminobenzidene tetrachloride as the chromogen. These slides had been carefully classified as showing villitis or not by three experienced pathologists separately on two occasions and in a third conjoint viewing. Sections were immunostained by overnight incubation with a 1:3000 dilution of a monoclonal antibody recognising the monomorphic determinants of the MHC class II antigens (CR3/ 43, Dako, Denmark). Negative controls were performed by replacing the primary antibody with normal goat serum and the use of irrelevant antibodies. A positive control on tonsil was run with each batch of immunostaining. The entirety of each slide was examined regardless of whether villitis was focal or diffuse, or absent.

\section{Results}

MHC class II immunoreactivity by villous components was seen in 17 of the 18 cases considered to have villitis by a consensus viewing by 
the three pathologists in the observer reliability study using conventional light microscopy (results not illustrated). Immunoreactivity was seen in villi affected by villitis and in unaffected villi. However, villitis foci also showed absent immunostaining. The one negative case resulted because the relevant focus of villitis had cut through in the deeper immunostained section. Of the 32 cases thought not to have villitis by the pathologists, 17 showed MHC class II immunoreactivity of variable intensity. Some of the positivity was seen in areas adjacent to infarcts.

\section{Discussion}

Although differences in methodology may account partly for the differing results, this study does not confirm that MHC class II immunoreactivity labels for villitis exclusively. MHC class II immunoreactivity near to infarcts indicates activation of the resident stromal macrophages and confirms their role in scavenger functions. Immunoreactivity in non-villitis areas in cases diagnosed conventionally as villitis as well as in non-villitis cases suggests immunopathology, but the pathogenesis is unclear and could be the result of infection or allogeneic recognition reactions. The possibility that villitis is a marker of a maternal immune attack on the fetal allograft ${ }^{2}$ is supported by the finding of maternal inflammatory cells in VUA lesions. ${ }^{3}$ However, the alternative possibility of a fetal response to foreign antigens, maternal or infectious, should not be discounted. Firstly, MHC class II immunoreactivity by syncytiotrophoblast is analogous to aberrant expression by epithelium in graft versus host disease ${ }^{9}$; secondly, the majority of the intravillous inflammation is fetal in origin, the proportion of maternal cells in affected villi ranging between $30 \%$ and $54 \%^{3}$; and finally, in this study MHC class II immunoreactivity was detected in non-inflamed areas. Without double labelling experiments, it could not be determined whether the labelling in non-inflamed areas was present in syncytiotrophoblast and the resident stromal macrophages or confined to the latter. Labelling by syncytiotrophoblast could be the result of inducement by cytokines in the microenvironment and can lead to influx of maternal inflammatory cells and a reactive activation of resident fetal macrophages. Labelling by macrophages alone, on the other hand, could be due to activation by an as yet unidentified agent and can lead to induction of syncytiotrophoblast MHC class II labelling. Further studies will be necessary to determine the sequence and primary site of MHC class II expression in non-inflamed villi as this may help to explain the initiating factors in the causation of villitis and in our understanding of its clinical significance. We have not excluded the hypothesis that villitis is an immunopathological lesion; we have merely found that immunopathology, if defined by MHC class II immunoreactivity, is present also in villi showing no histological evidence of villitis.

This study also questions the assumption that immunohistochemistry provides an objectivity that conventional histological methods lack. VUA is defined initially by conventional light microscopy but there can be significant intraobserver and interobserver unreliability in its diagnosis, one reason being the difficulty in distinguishing between stromal hypercellularity of the resident macrophages and true inflammation. MHC class II immunoreactivity by villitis cases led to a putative specific marker for villitis. Immunohistochemistry could then conceivably assume a precedent role over conventional light microscopy in defining the lesion. This is a paradigm of the chicken and the egg: how should the diagnosis be made and which criteria take priority? Although immunohistochemistry is being used increasingly as an aid to histological diagnosis and to define pathological lesions, cellular phenotype, and histogenesis, a dilemma can arise when there is conflict between the light microsocopy and immunohistochemistry diagnoses. ${ }^{10}$ Unfortunately, attempts to resolve this using decision analysis and probability calculations may not apply always as illustrated by this study.

Mrs C Amery provided technical support and the study was supported by the Queen Victoria Hospital Pathology Special Purposes Fund. This paper was presented in abstract form to Society for Medical Research.

1 Russell P. Inflammatory lesions of the human placenta. III. The histopathology of villitis of unknown aetiology. Placenta 1980;1:227-44.

2 Labarrere CA. Allogeneic recognition and rejection reactions in the placenta. Am $\mathcal{F}$ Reprod Immunol 1989;21: 94-9.

3 Redline RW, Patterson P. Villitis of unknown etiology is associated with major infiltration of fetal tissue by maternal inflammatory cells. Am F Pathol 1993;143:473-9.

4 Labarrere CA, Faulk WP, McIntyre JA. Villitis in normal term human placentae: frequency of the lesion determined by monoclonal antibody to HLA-DR antigen. $\mathcal{F}$ Reprod Immunol 1989;16:127-35.

5 Labarrere CA, McIntyre JA, Faulk WP. Immunohistologic evidence that villitis in human normal term placentas is an immunologic lesion. Am $₹$ Obstet Gynecol 1990;162 516-22.

6 Labarrere CA, Esmon CT, Carson SD, Faulk WP. Concordant expression of tissue factor and Class II MHC antigens in $11: 309-18$.

7 Labarrere CA, Faulk WP. MHC Class II reactivity of human villous trophoblast in chronic inflammation of unvillous trophoblast in chronic inflammation of

8 Khong TY, Staples A, Moore L, Byard RW. Observer reliability in assessing villitis of unknown aetiology. 7 Clin Pathol 1993;46:208-10.

9 Appleton AL, Sviland L. Current thoughts on the pathogenesis of graft versus host disease. $\mathcal{F}$ Clin Pathol 1993;46: 785-9.

10 Cook HT. Decision analysis in immunohistochemistry. Lancet 1991;338:257. 